Added in proof: Determination of the breadth of the level for $\mathrm{Ag}$ (22 sec.). A solution of $\mathrm{AgNO}_{3}$ and an equivalent solution of $\mathrm{HNO}_{3}$ were used alternatively for the slowing down of neutrons in a vessel, the dimensions of which were large, compared with the mean free path of neutrons belonging to the level of $\mathrm{Ag}(22$ sec.). Comparing the intensities of silver and of rhodium resonance neutrons in these two cases, we observed that $\mathrm{AgNO}_{3}\left(1.0 \mathrm{gm} . / \mathrm{cm} .^{3}\right)$ reduced the intensity of the neutrons belonging to the level $\mathrm{Ag}(22$ sec.) to 46 per cent, those belonging to the level Rh (45 sec.) only to 84 per cent of the intensity obtained in $\mathrm{HNO}_{3}$. Since the neutrons of the rhodium level are slower, this shows that only every fourth neutron becomes a silver neutron whilst being slowed down. The neutrons are not slowed down continuously, they lose on the average half their energy at every shock with a free proton. A resonance level is therefore only passed by all neutrons when its breadth is at least half its energy. Our result shows that three quarters of the neutrons jump over the silver level. Its breadth must therefore be $1 / 8$ of its energy or 0.4 volt. Of course 0.4 volt is at the same time the maximum breadth of the $\gamma$-ray of some millions of volts which is emitted when the neutron is captured.

We wish to thank Mr. P. Savitch for his efficient help and for interesting discussions. \section{Hans von HaLban, jun.} Peter Preiswerk.

\section{Institut du Radium, \\ Laboratoire Curie,} Paris.

\section{April 3.}

${ }^{2}$ P. Preiswerk and H. von Halban, jun., C.R., 202, 840 (1936).

2 O. R. Frisch and G. Placzek, NATURE, 137, 357 (1936).

3 Pontecorvo and Wick, La Ricerca Scientifica ii, 2, n.3-4.

‘ G. v. Hevesy and H. Levi, NATURe, 136, 103 (1936).

\section{Structure of Neutrons and $\beta$-Disintegration}

IT seems that an electron does not exist as a separate dynamical entity in a neutron. We assume, accordingly, that when a neutron is formed from a proton and an electron, the excess of energy of the particles is transformed into rest mass, energy and momentum being conserved.

If $\mathbf{u}, \mathbf{V}, \mathbf{V}^{\prime}$ are the velocities of an electron, proton and neutron, and $m, M, M^{\prime}$ their respective masses, then

$$
\begin{aligned}
& \frac{m \mathbf{u}}{\sqrt{ }\left(1-u^{2} / c^{2}\right)}+\frac{M \mathbf{V}}{\sqrt{ }\left(1-V^{2} / c^{2}\right)}=\frac{M^{\prime} \mathbf{V}^{\prime}}{\sqrt{ }\left(1-V^{\prime 2} / c^{2}\right)}+2 m \vec{m}, \\
& \frac{m}{\sqrt{ }\left(1-u^{2} / c^{2}\right)}+\frac{M}{\sqrt{ }\left(1-V^{2} / c^{2}\right)}=\frac{M^{\prime}}{\sqrt{ }\left(1-V^{\prime 2} / c^{2}\right)}+2 m ;
\end{aligned}
$$

where $2 m c^{2}$ is the binding energy.

Neglecting $V$, we find :

$$
M^{\prime} \equiv M-m+\left[\frac{m}{\sqrt{ }\left(1-u^{2} / c^{2}\right)}-m\right]
$$

the term in square brackets would correspond to the neutrino.

It would seem that the kinetic energy of $\beta$-rays, emitted at disintegration, is acquired at the expense of the rest mass of a neutron ; the difference between the masses of neutrons should, accordingly, be of the order of magnitude of the energy of $\beta$-rays.

Several values have been found for the mass of a neutron. For example, Chadwick and Curie-Joliot obtained 1.0067 and $1 \cdot 010$ respectively (difference about $6 m c^{2}$ or $3 \times 10^{6}$ volts). If the difference between the masses is genuine, and not due merely to experimental errors, it could be explained by the structure of neutrons suggested. It is not necessary to assume that the electron spin is destroyed at the formation of a neutron; it might remain unob. servable.

A classical picture of a neutron would be a rotating 'sphere' smoothly connected with a 'gyroscope' so that the spin of the latter remains uninfluenced by the external field.

Department of Mathematics,
Imperial College of Science,
S.W.7.

D. Meksyn.

\section{Thermal Decomposition of Acetaldehyde and Ethylene Oxides: Existence of Short-Lived Intermediates}

THE writer of the section of the Annual Reports of the Chemical Society, which has just been published, tells us that the results of the investigations of Hinshelwood and his co-workers on one hand, and of myself and my co-workers on the other, lead to the conclusion that the decomposition of acetaldehyde is influenced by chain mechanism at low temperatures but not at high temperatures. Whether the conclusion could justly be drawn from the published information available to the writer of the section of the Report is a matter of opinion; but it appears to be negatived by the results of more recent work.

Our work shows that, considered from the point of view of organic chemistry, when acetaldehyde is heated to temperatures between $360^{\circ}$ and $500^{\circ}$, and over a wide range of concentrations, decomposition follows two alternative courses, represented by the equations :

$$
\begin{aligned}
& \text { (1) } \mathrm{C}_{2} \mathrm{H}_{4} \mathrm{O} \\
& \text { or (2) } 2 \mathrm{C}_{2} \mathrm{H}_{4} \mathrm{O} \\
& =\mathrm{CH}_{4}+\mathrm{CO} \text {, } \\
& =\mathrm{CH}_{3}-\mathrm{CH}=\mathrm{CH}- \\
& \mathrm{CHO}+\mathrm{H}_{2} \mathrm{O} \text {, } \\
& \mathrm{CH}_{3}-\mathrm{CH}=\mathrm{CH}-\mathrm{CHO}=\mathrm{CH}_{3}-\mathrm{CH}=\mathrm{CH}_{2}+ \\
& \text { CO. }
\end{aligned}
$$

The former process is dominant in an unpacked tube, though some propylene is always formed; but the second process is dominant in a packed tube. It may be added that the propylene always undergoes some condensation, with the result that some 6-carbon hydrocarbon is produced, together with more com. plex products.

Now it would appear at first sight that we are dealing with two quite independent processes, one taking place on the surface, and the other in the gas phase; and as the volume change accompanying the second set of changes is relatively only a little less than that accompanying the simpler process represented by the first equation, the extent to which each of the processes takes place cannot be determined from the results of pressure measurements. Detailed analytical methods do, however, enable us to follow the processes exactly, and when this method is adopted we arrive at a very interesting conclusion.

While packing a reaction tube results in the slowing down of the first process and accelerating the second, it is found that the total rate of decomposition of the acetaldehyde is altogether independent of the nature or dimensions of the reaction tube. One must conclude therefore that the first stage in the process of decomposition, over the range of temperature 